\title{
Cesium Cs-131
}

National Cancer Institute

\section{Source}

National Cancer Institute. Cesium Cs-131. NCI Thesaurus. Code C95022.

An unstable radioisotope of cesium (Cs) with radiocytotoxic application. Cs-131 is a gamma photon-emitting radionuclide with high energy and a relatively short half-life of 9.7 days. When used in prostate brachytherapy, Cs-131 demonstrated advantages over other commonly used isotopes. 